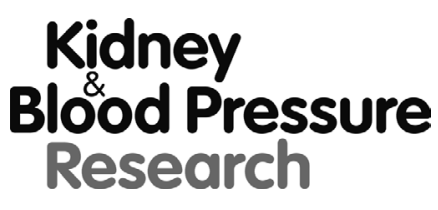

Original Paper

\begin{tabular}{l|l}
\hline \multicolumn{2}{l|}{ Kidney Blood Press Res 2018;43:1573-1584 } \\
\hline DOI: 10.1159/000494443 & $\begin{array}{l}\text { ○ 2018 The Author(s) } \\
\text { Published by S. Karger AG, Basel } \\
\text { Published online: 22 October } 2018\end{array}$ \\
Accepted: 12 October 2018 & \\
\hline
\end{tabular}

\title{
Peritonitis: Episode Sequence, Microbiological Variation, Risk Factors and Clinical Outcomes in a North China Peritoneal Dialysis Center
}

\author{
Shouci Hu ${ }^{\mathrm{a}}$ Pei Ming ${ }^{\mathrm{a}}$ Abdul Rashid Qureshib ${ }^{\mathrm{b}}$ Bengt Lindholm ${ }^{\mathrm{b}}$ Yang Bo \\ Hongtao Yang a \\ aDivision of Nephrology, First Affiliated Teaching Hospital, Tianjin University of Traditional Chinese \\ Medicine, Tianjin, China, 'Division of Renal Medicine and Baxter Novum, Department of Clinical \\ Science, Intervention and Technology, Karolinska Institutet, Stockholm, Sweden
}

\section{Key Words}

Peritoneal dialysis $•$ Peritonitis $・$ Microbiology $•$ Clinical outcomes

\begin{abstract}
Background/Aims: This study investigated peritonitis episodes with regard to time sequence, microbiological variation, factors associated with peritonitis and clinical outcomes in peritoneal dialysis (PD) patients. Methods: This single-center cohort study enrolled all incident patients who met the inclusion criteria at our center from June 1, 2012 to June 30, 2015 and who were followed until June, 2017. Clinical, biochemical characteristics and detailed data on peritonitis episodes, and hospitalizations were recorded. Results: A total of 218 episodes of peritonitis corresponding to a rate of 0.27 episode per patient-year were recorded. Gram positive bacteria, identified in 115 (52.8\%) episodes, were the most common pathogens. The occurrence of enterococcus peritonitis increased from $15.1 \%$ of the first to $27.3 \%$ of the later episodes. Multivariate logistic regression showed that the presence of cardiovascular disease (CVD, odds ratio $[\mathrm{OR}] 2.177,95 \%$ confidence interval $[95 \% \mathrm{Cl}] 1.214-3.903, \mathrm{P}=0.009)$, age $\leq$ 55 (OR 2.282, 95\% Cl 1.062-4.906, $\mathrm{P}=0.035)$, non-independent operator (OR $0.440,95 \% \mathrm{Cl}$ $0.206-0.938, P=0.034)$, lower values of potassium $(\mathrm{OR}=0.671,95 \% \mathrm{Cl} 0.472-0.954, \mathrm{P}=0.026)$ and higher values of calcium-phosphate product (OR 1.410,95\% $\mathrm{Cl} 1.065-1.868, \mathrm{P}=0.017$ ) were associated with peritonitis. Besides CVD (risk ratio [RR] 2.591, 95\%Cl 1.893-3.543, $\mathrm{P}<0.001$ ) and non-independent operator (RR $0.583,95 \% \mathrm{Cl} 0.439-0.776, \mathrm{P}<0.001)$, a lower level of education ( $R R \quad 0.641,95 \% \mathrm{Cl} 0.487-0.842, \mathrm{P}=0.001$ ) was associated with higher peritonitis rates in log-linear analysis. Spearman analyses indicated that the time to the 1 st episode was negatively related to the peritonitis rate $(r=-0.291, P=0.001)$. Time-dependent Cox regression showed no association between the time to the 1 st episode and patient survival $(P=0.151)$. Patients with a high peritonitis rate $(H P R)$ demonstrated worse technique survival $(P<0.001)$.




\section{Kidney Blood Pressure Research}

Conclusion: The present study has revealed several center-based features and modifiable risk factors for peritonitis. The presence of CVD and the need for assistance with PD operation not only increased the odds of peritonitis but were also associated with more peritonitis episodes. Time to first peritonitis was related to the peritonitis rate but not associated with patient survival. Patients with HPR had worse technique survival.

\section{Introduction}

With the continuing technical progress and improvement in clinical outcomes, peritoneal dialysis (PD) has become a high quality and cost-effective dialysis modality and is currently a main renal replacement therapy (RRT) for the treatment of end-stage kidney disease (ESKD). The past decade has witnessed the greatest increase in PD utilization in China, Thailand, and the USA [1]. However, as the prevalence of ESKD continues to rise [2], so does the need for improving the delivery, efficacy and safety of PD. PD-related peritonitis, which is a common yet important complication of PD, remains a major cause of hospital admission and cessation of PD, resulting in higher morbidity and mortality [3-5].

For a successful PD program, the prevention and management of peritonitis are deemed crucial. An increasing body of studies have focused on the first occurrence of peritonitis, especially the time to the first episode, which is most useful when identifying patients who are peritonitis-free and for measuring early-onset peritonitis [6-8]. Considerable variations in the peritonitis rate, as well as peritonitis outcomes, have been observed across centers, regions, countries, and races [9-12]. Although not well-established, it has been reported that patient characteristics, practice patterns, and socioeconomic status play a role in these disparities [13-15].

On an exploratory basis, in the present study, we retrospectively reviewed incident PD patients for a period of 5 years and mainly focused on the occurrence and impact of peritonitis, variations in microbiology, and changing episodes over time, factors that are associated with clinical outcomes. By investigating center-based infection patterns and risk factors, the present study provides evidence to improve the quality of PD care delivery.

\section{Materials and Methods}

This was a single-center, cohort study of all incident patients who used PD as their first RRT modality in our PD center from June 1, 2012 to June 30, 2015. Inclusion criteria were patients with ESKD who were aged $\geq 18$ years at the start of PD and who initiated PD therapy and were followed up at the PD center of the First Teaching Hospital of Tianjin University of Traditional Chinese Medicine, and were stable on PD therapy for more than 90 days; patients with missing baseline information, signs of ineligibility for PD modality, unwillingness to participate, who were transferred from permanent HD ( $\geq 3$ months), or following failed renal transplantation were excluded. The enrolled patients were recruited, and followed up until cessation of PD, death or June 30, 2017.

During June 1, 2012 to June 30, 2015, a total of 308 incident patients received PD catheter insertions with open surgical techniques at our PD center and none of these patients had a history of HD therapy for more than 3 months or graft failure. Among them, 15 patients dropped within the first 90 days (peritonitis was involved in 2 cases: one died with unresolved peritonitis, and the other switched to HD due to fungal peritonitis), 8 patients refused to be followed-up after catheter insertion, and 7 patients had missing basic information. Thus, a total of 278 patients ranging in age from 19 to 91 years who met the inclusion criteria were enrolled in this study and were followed for a median 33 (IQR, 25-45) months up to 5 years through June, 2017. Continuous ambulatory peritoneal dialysis (CAPD) modality was used on all patients. Conventional PD solutions (1.5\%, 2.5\%, or 4.25\% dextrose) and Y connections with double-bag systems were utilized in all the CAPD patients. This study was registered with the Chinese Clinical Trial Registry (ChiCTR-ORC-17013824) and was conducted in adherence to the declaration of Helsinki. The protocol of this 


\section{Kidney Blood Pressure Research}

study was approved by the Chinese Ethics Committee of Registering Clinical Trial (ChiECRCT-20170089), and informed consent was exempted because only aggregated data were received. The patient information was anonymized and de-identified prior to analysis.

\section{Data collection}

The data were obtained from the PD center database of the First Teaching Hospital of Tianjin University of Traditional Chinese Medicine. Information on all patients receiving PD was initially collected every year. Peritonitis episodes, hospital admissions, and detailed causes for the cessation of PD were recorded from the clinical charts. Baseline demographic data comprised of age, gender, PD inception date, marital status, educational degree, performer of home PD (patient or assistant), primary cause of ESKD, mean arterial pressure (MAP), comorbidities (hypertension, diabetes, foot ulcers, and CVD including coronary artery disease, myocardial infarction, congestive heart failure, cerebrovascular disease, stroke, atrial fibrillation, or peripheral arterial disease) were recorded at the initiation of PD. Clinical and biochemical data included body mass index (BMI), potassium, corrected calcium, phosphorus, calcium-phosphate product (Ca $\times \mathrm{P})$, intact parathyroid hormone (iPTH), high-sensitivity C-reactive protein (hs-CRP), serum albumin, glucose, creatinine, urea nitrogen, triglyceride, hemoglobin and ferritin. Dialysis data, including weekly total Kt/V urea, creatinine clearance $(\mathrm{CrCl})$, residual kidney function $(\mathrm{RKF})$, normalized protein catabolic rate (nPCR), $24 \mathrm{~h}$ urine output and ultrafiltration were also recorded. $4 \mathrm{~h}$ dialysate-to-plasma ratio of creatinine (D/Pcr) was measured by a standard peritoneal equilibration test.

Baseline biochemical data were collected within the first 1-3 months, and dialysis adequacy data were collected within the first 6 months after PD initiation. The total number of peritonitis episodes, hospital admissions due to non-peritonitis-related causes and cardiovascular events, and dates of the first peritonitis episode were recorded, where recorded episodes of peritonitis met at least 2 of the following 3 criteria: 1) clinical features of peritonitis; 2) dialysis effluent white cell count $>0.1 \times 109 / \mathrm{L}$ with $>50 \%$ polymorphonuclear; 3) positive dialysis effluent culture; according to the 2016 International Society for Peritoneal Dialysis (ISPD) recommendations [5], and the first peritonitis episode was defined as the first case since the initiation of PD where the criteria noted above were met. Peritonitis rates and hospitalization rates were expressed as events per patient-year.

Microbiology procedures including isolates identification, and susceptibility testing were performed in the Lab unit, First Teaching Hospital of Tianjin University of Traditional Chinese Medicine, with methodology and results interpreted according to the national clinical laboratory standard operation procedure and the criteria of the Clinical and Laboratory Standards Institute [16]. The terminology set of "relapsing, recurrent, and repeat peritonitis" as defined by ISPD [5] was applied to the present study. The management of peritonitis including empiric antibiotic selection, antibiotics regimen, adjunctive treatments and catheter removal was based on the patients' clinical status and adjusted for culture results and sensitivities, according to the guidelines of ISPD in combination with our center's experience.

\section{Statistical methods}

Median (10-90 percentile) or mean \pm standard deviation or percentage was determined for patient baseline demographics, laboratory parameters, and outcomes when appropriate. Differences between peritonitis and peritonitis-free groups were evaluated by Student's t-test for parametric data, the MannWhitney test or Kruskal-Wallis test for nonparametric data and the chi-square test ( $\chi 2)$ or Fisher's exact test for comparisons of percentages between groups, as appropriate. Event rates (per patient-year) were calculated for peritonitis episodes, non-peritonitis-related hospital admissions and cardiovascular hospitalization events. Survival curves were generated by the Kaplan-Meier method and compared by the log-rank test for patients dichotomized by the peritonitis status (with or without peritonitis episodes) during the first 24 months and by the median peritonitis rate of the peritonitis group (over or below). Cumulative proportional survival rates of peritonitis-free were derived from life table analysis. The censored events included all-cause death, switching to HD, renal transplantation, loss to follow-up or still active on PD at our center on June 30, 2017, with the exception of all-cause death for patient survival and switching to HD or died due to PD-related complications for technique survival. As the time to peritonitis event can be effected by PD duration, thus Cox regression model with a time-dependent covariate was applied to test the association between the time to 1st episode and patient survival by using the following logical expressions: 


\section{Kidney Blood Pressure Research}

$\mathrm{T}_{-} \mathrm{COV}_{-}=\left(\mathrm{T}_{-}<\right.$time to 1 st episode $\mid$ time to 1 st episode $\left.=9999\right) * 0+\left(\mathrm{T}_{-}>=\right.$time to 1 st episode $) * 1$, where "time to 1st episode" for peritonitis-free patients was set to be 9999 . The correlations between "time to 1st episode" and peritonitis rate, as well as "time to 1st episode" and "time on dialysis", were tested by Spearman analysis.

Binary logistic regression was conducted to assess the risk factors for the occurrence of peritonitis in all patients. Gender, age $\leq 55$, diabetes mellitus, and covariates with $\mathrm{P}<0.2$ in univariate analyses were included in the final multivariate logistic regression using backward stepwise procedure with an entry criteria of $\mathrm{P}<$ 0.05. General log-linear analysis with Poisson distribution was applied to model the peritonitis rate and estimate the main effect of the related factors in patients with peritonitis episodes, using peritonitis episode counts to weight cases, and patient-year at risk as cell structure variable. The results were expressed as the odds ratio (OR) with 95\% confidence interval (CI) in the binary logistic model or risk ratio (RR) with 95\% CI (calculated by exponentiating values of the estimated coefficients) in log-linear analysis. Statistical analyses were performed using IBMC SPSS ${ }^{\circ}$ Statistics Version 25. A P-value $<0.05$ was considered statistically significant.

\section{Ethical approval}

The study protocol has been approved by the Chinese Ethics Committee of Registering Clinical Trial (ChiECRCT-20170089), and informed consent was exempted because only aggregated data were received. The patient information was anonymized and de-identified prior to analysis.

\section{Results}

Of a total of 278 enrolled incident PD patients, 136 (48.9\%) patients were identified as having at least 1 episode of peritonitis and thus categorized in the peritonitis group, while $142(51.1 \%)$ patients with no peritonitis episodes during the follow-up were classified into the peritonitis-free group. Additionally, 97 patients experienced the 1st peritonitis within 24 months after the initiation of $\mathrm{PD}$, while 181 patients were peritonitis-free during the first 24 months. The median (IQR) follow-up period was 39 (29-50) months for patients with peritonitis episodes and 29 (24-41) months for peritonitis-free patients. By the end of June 30,2017 , a total of 218 episodes of peritonitis were recorded, corresponding to a rate of 0.27 episodes per patient-year. The median peritonitis rate for the peritonitis group $(n=136)$ was 0.42 (95\%CI 0.38-0.49) per patient-year. The cumulative peritonitis-free survival was $75 \%$, $61 \%$, and $49 \%$ at 1,2 and 3 years, respectively. Table 1 shows the main baseline demographic and clinical characteristics of the study patients. Compared with peritonitis-free patients, a higher prevalence of CVD and higher laboratory values for corrected calcium and Ca×P were observed in patients with peritonitis at the baseline.

\section{First Episode of Peritonitis, Later Episodes, and Clinical Outcomes}

Of a total of 218 episodes of peritonitis, 136 cases represented the first peritonitis episode and 82 cases occurred afterwards; gram-positive organisms were identified in 115 $(52.8 \%)$ episodes, $38(17.4 \%)$ episodes were gram-negative, and $6(2.8 \%)$ episodes were fungal. Culture-negative results were reported in 56 (25.7\%) cases, and 3 cases had missing information on culture. Episodes of polymicrobial peritonitis were found in one case of multiple gram-positive peritonitis and 2 cases of fungal peritonitis mixed with Klebsiella oxytoca, and Enterococcus faecium, respectively. Specific causative organisms in the first and the later episodes are summarized in Fig. 1. Among all episodes of culture-positive peritonitis $(\mathrm{n}=159)$, the leading encountered agent was Staphylococcus epidermidis (31 cases), which accounted for $21.5 \%$ of the first and $16.7 \%$ of the later episodes. Enterococcus faecalis was the second most frequent cause (27 cases), resulting in $14.0 \%$ of the first and $21.2 \%$ of the later episodes. Escherichia coli (21 cases) was the main causative organism for gram-negative peritonitis. Among these prevalent organisms, the occurrence of enterococci fluctuated the most, increasing from $15.1 \%$ in the first to $27.3 \%$ in the later episodes. Vancomycin-resistant 


\section{Kidney Blood Pressure Research}

Table 1. Baseline Demographic and Clinical Characteristics for Incident PD Patients with and without Episodes of Peritonitis. Abbreviations: PD, peritoneal dialysis; ESKD, end stage kidney disease; MAP, mean arterial pressure; BMI, body mass index; iPTH, intact parathyroid hormone; hs-CRP, high-sensitivity C-reactive protein; Kt/V urea, urea kinetics; $\mathrm{CrCl}$, creatinine clearance; RKF, residual kidney function; $\mathrm{nPCR}$, normalized protein catabolic rate; D/Pcr, dialysate-to-plasma creatinine ratio. Boldface indicates $\mathrm{P}$ values less than 0.05 , which are considered statistically significant

\begin{tabular}{|c|c|c|c|c|c|}
\hline Variable & $\mathrm{n}$ & $\begin{array}{l}\text { Peritonitis group } \\
\text { median (10-90 percentile) } \\
\text { or } \mathrm{n}(\%)\end{array}$ & $\mathrm{n}$ & $\begin{array}{l}\text { Peritonitis-free group } \\
\text { median }(10-90 \text { percentile }) \\
\text { or } \mathrm{n}(\%)\end{array}$ & $\begin{array}{c}\mathrm{P} \\
\text { value }\end{array}$ \\
\hline Age (years) & 136 & $58(34-76)$ & 142 & $58(31-75)$ & 0.561 \\
\hline Female gender (\%) & 136 & 69 (50.7\%) & 142 & 69 (48.6\%) & 0.810 \\
\hline Married (\%) & 136 & $120(88.2 \%)$ & 142 & $126(88.7 \%)$ & 1.000 \\
\hline Education: senior high and & 136 & $53(39.0 \%)$ & 142 & $67(47.2 \%)$ & 0.184 \\
\hline Self-operator (\%) & 136 & $54(39.7 \%)$ & 142 & $69(48.6 \%)$ & 0.148 \\
\hline \multicolumn{6}{|l|}{ Primary cause for ESKD } \\
\hline Glomerulonephritis (\%) & 136 & $49(36.0 \%)$ & 142 & $49(34.5 \%)$ & \\
\hline Diabetic kidney disease (\%) & 136 & $49(36.0 \%)$ & 142 & $50(35.2 \%)$ & \\
\hline Hypertension (\%) & 136 & $4(2.9 \%)$ & 142 & $12(8.5 \%)$ & \\
\hline Polycystic kidney disease (\%) & 136 & $4(2.9 \%)$ & 142 & $3(2.1 \%)$ & \\
\hline Others $(\%)$ & 136 & $30(22.1 \%)$ & 142 & $28(19.7 \%)$ & \\
\hline Diabetes mellitus (\%) & 136 & $61(44.9 \%)$ & 142 & $56(39.4 \%)$ & 0.396 \\
\hline Cardiovascular disease (\%) & 136 & 106 (77.9\%) & 142 & $89(62.7 \%)$ & 0.006 \\
\hline MAP (mmHg) & 136 & $100(93-110)$ & 142 & $100(97-110)$ & 0.893 \\
\hline BMI $\left(\mathrm{kg} / \mathrm{m}^{2}\right)$ & 136 & $23.9(19.4-29.6)$ & 142 & $23.5(18.9-29.6)$ & 0.606 \\
\hline Potassium (mmol/L) & 136 & $4.1(3.2-5.2)$ & 140 & $4.2(3.3-5.2)$ & 0.169 \\
\hline Corrected calcium (mmol/L) & 136 & $2.30(2.08-2.58)$ & 140 & $2.26(1.96-2.50)$ & 0.007 \\
\hline Phosphorus (mmol/L) & 136 & $1.60(1.10-2.21)$ & 140 & $1.55(1.08-2.07)$ & 0.313 \\
\hline $\mathrm{Ca} \times \mathrm{P}\left(\mathrm{mmol}^{2} / \mathrm{L}^{2}\right)$ & 136 & $3.75(2.49-5.15)$ & 140 & $3.50(2.58-4.75)$ & 0.021 \\
\hline iPTH (ng/L) & 135 & $297.9(45.3-649.2)$ & 132 & $323.2(92.2-852.4)$ & 0.129 \\
\hline Triglyceride (mmol/L) & 136 & $1.54(0.79-3.32)$ & 140 & $1.66(0.90-3.10)$ & 0.391 \\
\hline Blood urea nitrogen (mmol/L) & 136 & $17.42(11.29-23.83)$ & 140 & 18.39 (11.84-24.53) & 0.361 \\
\hline Serum creatine $(\mu \mathrm{mmol} / \mathrm{L})$ & 136 & $608.4(396.8-979.4)$ & 140 & 619.1 (424.4-952.5) & 0.965 \\
\hline Serum glucose $(\mathrm{mmol} / \mathrm{L})$ & 136 & $5.5(4.4-8.8)$ & 140 & $5.3(4.3-8.0)$ & 0.109 \\
\hline Serum albumin $(\mathrm{g} / \mathrm{L})$ & 136 & $33.6(26.1-39.5)$ & 140 & $34.1(24.1-40.0)$ & 0.709 \\
\hline Hemoglobin (g/L) & 136 & $107(88-134)$ & 140 & $108(80-125)$ & 0.219 \\
\hline Ferritin $(\mu \mathrm{g} / \mathrm{L})$ & 135 & $144.7(38.5-496.6)$ & 132 & $130.1(28.3-420.5)$ & 0.060 \\
\hline hs-CRP (mg/L) & 125 & $3.2(3.1-28.7)$ & 122 & $3.3(3.1-21.0)$ & 0.289 \\
\hline $\mathrm{RKF}\left(\mathrm{mL} / \mathrm{min} / 1.73 \mathrm{~m}^{2}\right)$ & 129 & $2.76(0.65-6.72)$ & 122 & $2.88(0.56-6.38)$ & 0.858 \\
\hline Total Kt/V urea & 129 & $1.82(1.18-2.83)$ & 122 & $1.78(1.12-2.54)$ & 0.272 \\
\hline Total CrCl (l/week/1.73 m²) & 129 & $61.04(44.24-100.15)$ & 122 & 61.17 (39.05-91.98) & 0.908 \\
\hline Ultrafiltration (mL/day) & 129 & $600(75-1260)$ & 122 & 545 (33-1253) & 0.273 \\
\hline Urine output (mL/day) & 129 & $850(300-1700)$ & 122 & $900(200-1700)$ & 0.421 \\
\hline nPCR & 129 & $1.23(0.92-1.72)$ & 122 & $1.16(0.83-1.51)$ & 0.332 \\
\hline 4-h D/Pcr & 122 & $0.61(0.47-0.75)$ & 113 & $0.62(0.46-0.78)$ & 0.654 \\
\hline \multicolumn{6}{|l|}{ Peritoneal transport status } \\
\hline High + high average (\%) & 122 & $44(36.1 \%)$ & 113 & $44(38.9 \%)$ & 0.687 \\
\hline
\end{tabular}

enterococci (VRE) were found in 6 cases of enterococcus peritonitis (and an intermediate minimum inhibitory concentration range for vancomycin was reported in 2 cases).

The outcomes of the first episode of peritonitis according to different causative organism are provided in Table 2 . The complete cure, recurrent, relapsing and repeat rates for all first episodes were $82.4 \%, 5.1 \%, 1.5 \%$, and $2.9 \%$, respectively. Three of seven cases of catheter removal were fungal peritonitis, and a total of $4(2.9 \%)$ death cases occurred during unresolved peritonitis.

Table 3 shows the clinical outcomes for patients with and without episodes of peritonitis. The crude mortality rate for all incident PD patients was $21.9 \%$, cardiovascular death was accounting for $33.3 \%$ and $47.1 \%$ of mortality in peritonitis and peritonitis-free groups, respectively. Fifteen of 20 patients who switched to HD had experienced peritonitis while 16 of 23 patients who received kidney transplantation were peritonitis-free. Two hundred thirteen $(76.6 \%)$ patients were hospitalized at least once due to non-peritonitisrelated causes, and for patients with and without peritonitis, the non-peritonitis-related 


\section{Kidney Blood Pressure Research}

hospitalization rate was 0.91 and 0.79 per patient-year. Moreover, the cardiovascular hospitalization rates for the peritonitis group and peritonitis-free groups were 0.25 and 0.23 per patient-year, respectively.

\section{Factors Associated with Peritonitis}

Results from univariate logistic regression (shown in Fig. 2) indicated that, comorbid CVD $(\mathrm{P}=0.006)$, and a higher value for calcium-phosphate product $(\mathrm{P}=0.038)$ were factors associated with peritonitis. After adjusting for female gender, age $\leq 55$, diabetes, and all the covariates with $\mathrm{P}$ values $<0.2$, the final multivariate logistic regression as summarized in Table 4 indicated that comorbid CVD, age $\leq 55$, non-independent operator, lower values for potassium, and higher values for $\mathrm{Ca} \times \mathrm{P}$ were significantly associated with the occurrence of peritonitis. General loglinear analysis modelling of peritonitis rate (shown in Table 5) indicated that comorbid CVD, a lower level of education, and non-independent operator were significantly associated with higher peritonitis rates, while female gender, age $\leq 55$, and diabetes showed no statistical significance. Spearman analyses indicated that "time to 1st episode" was negatively related to peritonitis rate $(\mathrm{r}=-$ 0.291, $\mathrm{P}=0.001$ ).

Time to first Peritonitis, Patient Survival and Technique Survival

No statistical significance was observed from the time-dependent Cox regression when "time to 1st episode" was applied as a time covariate to model patient survival $(\mathrm{HR}=1.478,95 \% \mathrm{CI}$ 0.867-2.520, $\quad \mathrm{P}=0.151)$. Spearman analyses indicated that "time to 1 st episode" was positively related to PD duration $(\mathrm{r}=0.307$, $\left.\mathrm{P}<\begin{array}{llll}0 & 0 & 0 & 1\end{array}\right)$. Kaplan-Meie r survival curves for patients free from peritonitis in the first 24 months and patients who had records of peritonitis during the same time period are shown in Fig. 3a and Fig. 3b, but no significant difference was

Table 2. Outcomes for the 1st Peritonitis Episode

\begin{tabular}{lcccc}
\hline \multirow{2}{*}{ Outcomes } & Total & \multicolumn{3}{c}{ Organism of the 1st Episode of Peritonitis } \\
& $\mathrm{n}=136$ & Gram $+(\mathrm{n}=64)$ & Gram- $(\mathrm{n}=25)$ & Others (n=47) \\
\hline Cure & $112(82.4 \%)$ & $53(82.8 \%)$ & $22(88.0 \%)$ & $37(78.7 \%)$ \\
Recurrent & $7(5.1 \%)$ & $4(6.3 \%)$ & 0 & $3(6.4 \%)$ \\
Relapse & $2(1.5 \%)$ & 0 & $1(4.0 \%)$ & $1(2.1 \%)$ \\
Repeat & $4(2.9 \%)$ & $4(6.3 \%)$ & 0 & 0 \\
Catheter removal & $7(5.1 \%)$ & $1(1.6 \%)$ & $2(8.0 \%)$ & $4(8.5 \%)$ \\
Death (occurred during unresolved peritonitis) & $4(2.9 \%)$ & $2(3.1 \%)$ & 0 & $2(4.3 \%)$ \\
\hline
\end{tabular}

Table 3. Clinical Outcomes for Incident PD Patients with and without Episodes of Peritonitis. Abbreviations: PD, peritoneal dialysis; HD, hemodialysis

\begin{tabular}{lcc}
\hline Outcomes & $\begin{array}{c}\text { Peritonitis group } \\
(\mathrm{n}=136)\end{array}$ & $\begin{array}{c}\text { Peritonitis-free group } \\
(\mathrm{n}=142)\end{array}$ \\
\hline PD duration (mean \pm SD, months) & $38.3 \pm 13.3$ & $31.9 \pm 13.0$ \\
All-cause death, N (\%) & $27(19.9 \%)$ & $34(23.9 \%)$ \\
- Cardiovascular Death, N (\%) & $9(33.3 \%)$ & $16(47.1 \%)$ \\
Transplantation, N (\%) & $7(5.1 \%)$ & $16(11.3 \%)$ \\
Switched to HD, N (\%) & $15(11.0 \%)$ & $5(3.5 \%)$ \\
Non-peritonitis-related hospital admission, N (\%) & $109(80.1 \%)$ & $104(73.2 \%)$ \\
Cardiovascular hospitalization, N (\%) & $54(39.7 \%)$ & $44(31.0 \%)$ \\
Non-peritonitis-related hospitalization rate (per patient- & 0.91 & 0.79 \\
Cardiovascular hospitalization rate (per patient-year) & 0.25 & 0.23 \\
\hline
\end{tabular}




\section{Kidney Blood Pressure Research}

found in patient survival (log rank $0.462, \mathrm{P}=0.497$ ) or technique survival (log rank 2.630, $\mathrm{P}=0.105$ ). Fig. $3 \mathrm{c}$ and Fig. $3 \mathrm{~d}$ show survival curves for patients with peritonitis rates over 0.42 per patientyear (high peritonitis rate, HPR) vs. patients with peritonitis rates below 0.42 per patient-year (low peritonitis rate, LPR). When compared with the LPR group, the HPR group showed worse technique survival (log rank 17.910, $\mathrm{P}=0.000$ ) but a comparable patient survival (log rank $0.357, \mathrm{P}=0.550$ ).

\section{Discussion}

With almost half $(48.9 \%)$ of the incident PD patients experiencing peritonitis during a period of 5 years, our study found a peritonitis rate of 0.27 per patient-year and cumulative peritonitis-free survival of $75 \%, 61 \%$, and $49 \%$ at 1,2 , and 3 years, respectively, in this cohort of 278 patients. A previous study conducted in a southern China center reported higher peritonitisfree survival, demonstrating $86.2 \%, 78.1 \%$, and $71.4 \%$ at 1,2 and 3 years, respectively [17]. Patients experiencing peritonitis had a higher burden of CVD and worse calcium and phosphate abnormalities at the baseline compared with peritonitis-free patients. S. epidermidis and E. faecalis for gram positive peritonitis and E. coli for gram negative peritonitis, were the most prevalent pathogens in our center. Consistent with the results from recent studies [8, $9,18]$, S. epidermidis was the main gram-positive bacterium causing peritonitis, and most gram-negative episodes were attributable to E. coli. S. epidermidis is mostly seen in cases of touch contamination, suggesting an urgent need of to improve the quality of patient training and retraining on aseptic technique $[4,5]$. Another concerning finding is the rising trend of enterococcus peritonitis and the occurrence of VRE, indicating that more attention should be paid to gastrointestinal problems and the review of bacterial sensitivity patterns.

Fig. 2. Univariate Logistic Regression Model in All Incident PD Patients $(\mathrm{n}=278)$. Binary Dependent Variable: Peritonitis (1), Peritonitis-free (0). CVD, cardiovascular disease; Kt/V urea, urea kinetics.

Table 4. Multivariable Logistic Regression Models (Backward Stepwise LR) in All Incident PD Patients ( $n=278)$. Binary Dependent Variable: Peritonitis (1), Peritonitis-free (0). a. Variables removed on steps 2, 3, and 4 were Diabetes mellitus, Male gender, and Higher level of education, respectively. Abbreviations: PD, peritoneal dialysis; CVD, cardiovascular disease; Kt/V urea, urea kinetics; LR, likelihood ratio; OR, odds ratio; $\mathrm{CI}$, confidence interval

\begin{tabular}{lcccc}
\hline Hosmer-Lemeshow Test & Variable & OR & $95 \% \mathrm{CI}$ & $\mathrm{P}$ \\
\hline Step 4a, P=0.657 & Comorbid CVD & 2.177 & $1.214-3.903$ & 0.009 \\
& Self-operator & 0.440 & $0.206-0.938$ & 0.034 \\
& Below the age of 55 & 2.282 & $1.062-4.906$ & 0.035 \\
& Potassium, per 1-mmol/L greater & 0.671 & $0.472-0.954$ & 0.026 \\
& CaxP, per 1-mmol $/ \mathrm{L}^{2}$ greater & 1.410 & $1.065-1.868$ & 0.017 \\
\hline
\end{tabular}

Table 5. General Loglinear Analysis (Poisson Distribution) for Incident PD Patients with Peritonitis $(n=136)$. Total Peritonitis Episodes: 218. Abbreviations: PD, peritoneal dialysis; CVD, cardiovascular disease; RR, risk ratio; $\mathrm{CI}$, confidence interval. Boldface indicates $\mathrm{P}$ values less than 0.05 considered to be statistically significant

\begin{tabular}{lcccc}
\hline Variable & Case Counts N (\%) & RR & $95 \%$ CI & P \\
\hline Female gender & $111(50.9 \%)$ & 0.989 & $0.757-1.293$ & 0.937 \\
Below the age of 55 & $102(46.8 \%)$ & 0.968 & $0.736-1.273$ & 0.814 \\
Comorbid CVD & $166(76.1 \%)$ & 2.591 & $1.893-3.543$ & $<0.001$ \\
Diabetes mellitus & $100(45.9 \%)$ & 0.813 & $0.616-1.074$ & 0.144 \\
Education: Senior high and above & $85(39.0 \%)$ & 0.641 & $0.487-0.842$ & 0.001 \\
Self-operator & $84(38.5 \%)$ & 0.583 & $0.439-0.776$ & $<0.001$ \\
\hline
\end{tabular}




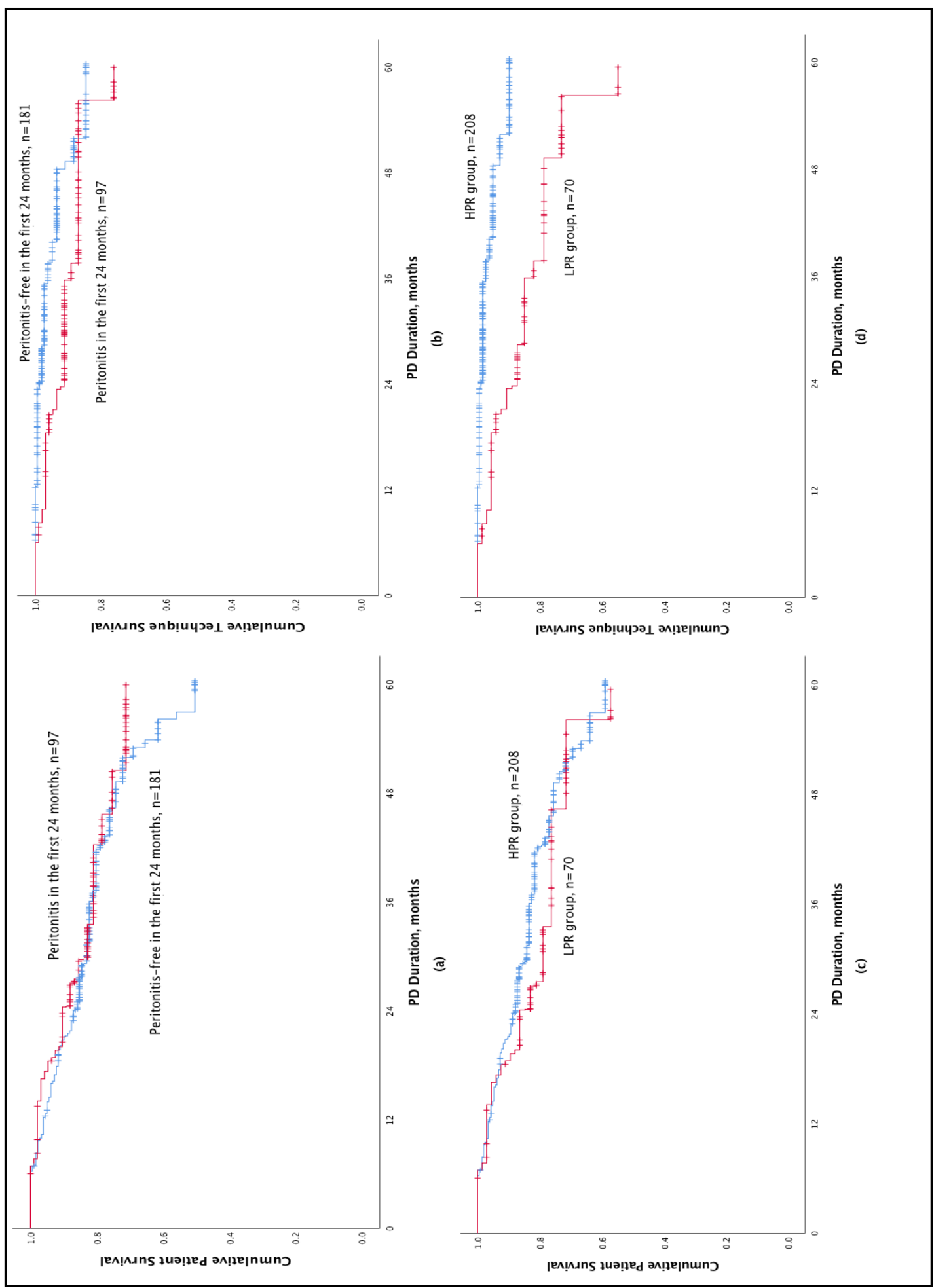

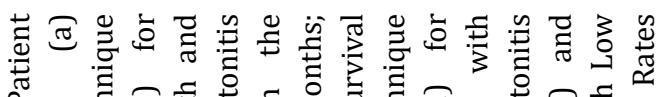

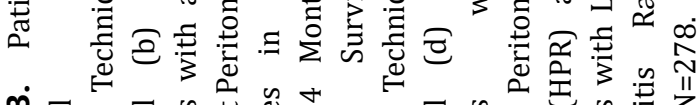
m

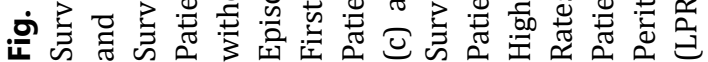




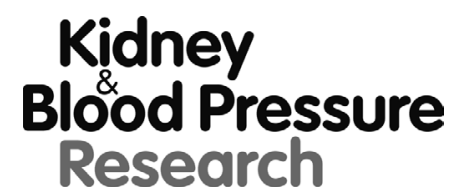
\begin{tabular}{l|l}
\hline Kidney Blood Press Res 2018;43:1573-1584 \\
\hline $\begin{array}{l}\text { DOI: } 10.1159 / 000494443 \\
\text { Published online: } 22 \text { October } 2018\end{array}$ & $\begin{array}{l}\text { c } 2018 \text { The Author(s). Published by S. Karger AG, Basel } \\
\text { www.karger.com/kbr }\end{array}$ \\
\hline
\end{tabular}

Hu et al.: Peritonitis and Risk Factors in Peritoneal Dialysis Patients

For the treatment of enterococcus peritonitis, ISPD guidelines recommend intra-peritoneal (IP) vancomycin. However, VRE infection has increased rapidly in recent years [19], optimal treatments remain controversial and limited. A study from Japan reported oral amoxicillin is an efficient and convenient substitute [20], and linezolid has been proven effective for VRE peritonitis $[21,22]$.

Despite a relatively high proportion of peritonitis patients, complete cures were achieved for most first peritonitis episodes, and the rates of recurrence, relapse, and repeat were comparatively low. Our study demonstrated comparable patient survival between patients with higher and lower rates of peritonitis, yet patients with HPR had worse technique survival. Of the patients who switched to permanent HD, 75\% had experienced peritonitis, and the relatively high rate of hospital admissions in patients with peritonitis episodes also implies an adverse effect from peritonitis.

Our study showed that patients with CVD, lower values of potassium, and calcium and phosphate abnormalities, and those in need of assistance with PD performance and aged below 55 had higher odds of peritonitis occurrence. In addition to CVD and assisted PD, a lower level of education was associated with more peritonitis episodes. Associations between peritonitis and coronary artery disease, cerebrovascular disease, or peripheral arterial disease have been reported in other studies $[4,6,23]$. Hypokalemia, which is considered to be related to impaired bowel motility and bacterial overgrowth [24], potentially poses a risk for peritonitis, and lower level of potassium has been consistently supported by several studies as a risk factor for peritonitis $[17,25,26]$. Calcium metabolism has been studied relatively little in peritonitis. Nevertheless, mineral and bone disorders can have an impact, especially when uremic pruritus [27] or fractures weigh in, causing touch contamination and unqualified PD performance. Kerschbaum et al. [28] reported that oral administration of active vitamin $\mathrm{D}$ is associated with a lower risk of peritonitis and according to a recent meta-analysis, the use of vitamin $\mathrm{D}$ in long-term dialysis patients appears to be associated with lower risk of infection-related outcomes [29]. The effect of educational attainment on peritonitis risk has been discussed in several studies; lower educational level and lower socioeconomic status $[3,6,17,14]$ are associated with higher peritonitis rates. One of our findings was at odds with previous studies in which advanced age is associated with peritonitis [30,31]. As a home-based dialysis modality, PD provides more self-managed time and space. Especially for younger patients, increased daily activities may expand the exposure to peritonitis risks, however, along with the other findings, we conclude that PD training and educational programs are of paramount importance for both patients and caregivers involved.

Interestingly, a handful of studies have reported that the time interval to first peritonitis influences clinical outcomes and peritonitis rates [8, 32-34]. In the present study, we found that the time to the first episode was positively related to PD duration and inversely related to the peritonitis rate but not associated with patient survival. Another study from Taiwan observed a "peritonitis paradox" when comparing the survival of patients with and without peritonitis by using the Kaplan-Meier method and log rank tests; patients who were peritonitis-free tended to do worse [30]. To avoid misleading results, in the present study, we selected the peritonitis status during the first 24 months since PD inception for comparison, and neither patient survival nor technique survival were different for patients with and without episodes of peritonitis during the first 24 months.

There are certain limitations in this study. First, as a single-center retrospective study with a limited number of patients and observed events, ascertainment bias, and type two errors cannot be avoided and the results can be neither generalized to all patients nor prove causation. Second, since patients who didn't survive the first 90 days were excluded from this study, there is possible selection bias. Third, the analytical methodology of this study has pitfalls as we were unable to exclude residual confounders, and only baseline data were used for multivariate analysis. However, to our knowledge, there are few studies regarding peritonitis episodes on a time sequence and our study has spotted a few modifiable 


\section{Kidney Blood Pressure Research}

peritonitis risk factors. Further prospective studies dealing with improving peritonitis rates are warranted.

\section{Conclusion}

Peritonitis episodes were mostly caused by Gram-positive organisms relating to touch contamination, and there was a rising trend of enterococcus peritonitis in our center. Patients with CVD, lower values of potassium, calcium and phosphate abnormalities and those in need of assistance for PD operation and aged below 55 had higher odds of peritonitis. A lower educational level, the presence of CVD, and assistance with PD operation were associated with higher peritonitis rates. The time interval to the first occurrence of peritonitis was related to the peritonitis rate and PD duration but not associated with patient survival. Patients with HPR had worse technique survival, but among patients with and without peritonitis occurrence in the first 2 years on PD, patient survival and technique survival were comparable.

\section{Acknowledgements}

We would like to thank all the hard-working doctors and nurses in our PD center for their excellent medical care and the assistance in data collection.

This work was supported by the National Natural Science Foundation of China (Grant No. 81373851) and Tianjin Science and Technology Program (Grant No. 15ZXLCSY00020).

The specific work of each author in this study was as follows:

Shouci Hu: conceptualization, data collection, data curation, formal analysis, data interpretation, writing (including draft, review and editing) and finalized the manuscript. Pei Ming: conceptualization, data collection, data curation, formal analysis, data interpretation, validation. Abdul Rashid Qureshi: conceptualization, formal analysis, data interpretation, validation. Bengt Lindholm: data interpretation, validation, manuscript editing. Yang Bo: data acquisition, data interpretation, validation. Hongtao Yang: conceptualization, data acquisition, validation, resources, supervision, final approval of the version to be published.

\section{Disclosure Statement}

Bengt Lindholm is employed by Baxter Healthcare and none of the other authors declare any conflict of interest.

\section{References}

$>1$ Li PKT, Chow KM, Luijtgaarden MWM Van de, Johnson DW, Jager KJ, Mehrotra R, Naicker S, Pecoits-Filho

$\mathrm{R}, \mathrm{Yu} \mathrm{XQ}$, Lameire N: Changes in the worldwide epidemiology of peritoneal dialysis. Nat Rev Nephrol 2017;13:90-103.

-2 Global Burden of Disease 2013 GFR Collaborators, CKD Prognosis Consortium, and Global Burden of Disease Genitourinary Expert Group: Global Cardiovascular and Renal Outcomes of Reduced GFR. J Am Soc Nephrol 2017;28:2167-2179.

3 Kumar VA, Sidell MA, Yang WT, Jones JP: Predictors of peritonitis, hospital days, and technique survival for peritoneal dialysis patients in a managed care setting. Perit Dial Int 2014;34:171-178.

4 Cho Y, Johnson DW: Peritoneal Dialysis-Related Peritonitis: Towards Improving Evidence, Practices, and Outcomes. Am J Kidney Dis 2014;64:278-289. 


\section{Kidney \\ Blood Pressure Research}

5 Li PK-T, Szeto CC, Piraino B, Arteaga J de, Fan S, Figueiredo AE, Fish DN, Goffin E, Kim YL, Salzer W, Struijk DG, Teitelbaum I, Johnson DW: ISPD peritonitis recommendations: 2016 update on prevention and treatment. Perit Dial Int 2016;36:481-508.

-6 See EJ, Johnson DW, Hawley CM, Pascoe EM, Darssan D, Clayton PA, Borlace M, Badve SV, Sud K, Boudville NC, Cho Y: Early peritonitis and its outcome in incident peritoneal dialysis patients. Perit Dial Int 2017; DOI:10.3747/pdi.2017.00029.

7 Vargas E, Blake PG, Sanabria M, Bunch A, López P, Vesga J, Buitrago A, Astudillo K, Devia M, Sánchez R: Early peritonitis in a large peritoneal dialysis provider system in colombia. Perit Dial Int 2017;37:30-34.

-8 Tian Y, Xie X, Xiang S, Yang X, Lin J, Zhang X, Shou Z, Chen J: Risk Factors and Outcomes of Early-Onset Peritonitis in Chinese Peritoneal Dialysis Patients. Kidney Blood Press Res 2017;6:1266-1276.

-9 Ong LM, Ch'ng CC, Wee HC, Supramaniam P, Zainal H, Goh BL, Bavanandan S, Mushahar L, Hooi LS, Ahmad G: Risk of peritoneal dialysis-related peritonitis in a multi-racial asian population. Perit Dial Int 2017;37:35-43.

10 See EJ, Johnson DW, Hawley CM, Pascoe EM, Badve SV, Boudville N, Clayton PA, Sud K, Polkinghorne KR, Borlace M, Cho Y: Risk Predictors and Causes of Technique Failure Within the First Year of Peritoneal Dialysis: An Australia and New Zealand Dialysis and Transplant Registry (ANZDATA) Study. Am J Kidney Dis 2017; DOI:10.1053/j.ajkd.2017.10.019.

11 Htay H, Cho Y, Pascoe EM, Darssan D, Hawley C, Clayton PA, Borlace M, Badve SV, Sud K, Boudville N, Mcdonald SP, Johnson DW: Center Effects and Peritoneal Dialysis Peritonitis Outcomes: Analysis of a National Registry. Am J Kidney Dis 2017; DOI:10.1053/j.ajkd.2017.10.017.

12 Ghali JR, Bannister KM, Brown FG, Rosman JB, Wiggins KJ, Johnson DW, McDonald SP: Microbiology and outcomes of peritonitis in Australian peritoneal dialysis patients. Perit Dial Int 2011;31:651-662.

13 Whitty R, Bargman JM, Kiss A, Dresser L, Lui P: Residual kidney function and peritoneal dialysis-associated peritonitis treatment outcomes. Clin J Am Soc Nephrol 2017;12:2016-2022.

-14 Tang W, Grace B, McDonald SP, Hawley CM, Badve SV, Boudville NC, Brown FG, Clayton PA, Johnson DW: Socio-economic status and peritonitis in Australian non-indigenous peritoneal dialysis patients. Perit Dial Int 2015;35:450-459.

15 Lanot A, Bechade C, Verger C, Fabre E, Vernier I, Lobbedez T: Clusters of practice in peritoneal dialysis in France: data from the catheter section of the RDPLF. Perit Dial Int 2017; DOI:10.3747/pdi.2017.00135.

16 Clinical and Laboratory Standards Institute: Performance Standards for Antimicrobial Susceptibility Testing; Twenty-Second Informational Supplement, M100-S22, 2012. URL: http://zums.ac.ir/files/health/ pages/ill/azmayeshghah/clsi_2013.pdf

17 Fan X, Huang R, Wang J, Ye H, Guo Q, Yi C, Lin J, Zhou Q, Shao F, Yu X, Yang X: Risk factors for the first episode of peritonitis in Southern Chinese continuous ambulatory peritoneal dialysis patients. PLoS One 2014;9:e107485.

18 Zelenitsky SA, Howarth J, Lagacé-Wiens P, Sathianathan C, Ariano R, Davis C, Verrelli M: Microbiological trends and antimicrobial resistance in peritoneal dialysis-related peritonitis, 2005 to 2014. Perit Dial Int 2017;37:170-176.

19 Murray BE: Vancomycin-resistant enterococcal infections. N Engl J Med 2000;342:710-721.

-20 Szeto CC, Ng JKC, Chow KM, Kwan BCH, Kwong VWK, Law MC, Leung CB, Li PKT: Treatment of enterococcal peritonitis in peritoneal dialysis patients by oral amoxicillin or intra-peritoneal vancomcyin: A retrospective study. Kidney Blood Press Res 2018;42:837-843.

-21 Song J, Seo JW, Kwon YE, Kim YL, Lim TS, Kang EW, Chang TI: Successful treatment of vancomycin-resistant enterococcus peritonitis using linezolid without catheter removal in a peritoneal dialysis patient. Perit Dial Int 2014;34:235-239.

22 Unal A, Agkus C, Kocyigit I, Oymak O, Utas C: Peritoneal dialysis-related peritonitis caused by vancomycinresistant Enterococcus faecium. Ther Apher Dial 2011;15:115-116.

23 Pulliam J, Li NC, Maddux F, Hakim R, Finkelstein FO, Lacson E: First-year outcomes of incident peritoneal dialysis patients in the United States. Am J Kidney Dis 2014;64:761-769.

24 Zanger R: Hyponatremia and Hypokalemia in Patients on Peritoneal Dialysis. Semin Dial 2010;23:575-580.

25 Chuang YW, Shu KH, Yu TM, Cheng CH, Chen CH: Hypokalaemia: An independent risk factor of enterobacteriaceae peritonitis in CAPD patients. Nephrol Dial Transplant 2009;24:1603-1608. 


\section{Kidney \\ Blood Pressure Research}

-26 Ribeiro SC, Figueiredo AE, Barretti P, Pecoits-Filho R, De Moraes, Thyago Proenca, all centers that contributed to the BRAZPD II study: Low serum potassium levels increase the infectious-caused mortality in peritoneal dialysis patients: A propensity-matched score study. PLoS One 2015;10:e0127453.

-27 Scherer JS, Combs SA, Brennan F: Sleep Disorders, Restless Legs Syndrome, and Uremic Pruritus: Diagnosis and Treatment of Common Symptoms in Dialysis Patients. Am J Kidney Dis 2017;69:117-128.

-28 Kerschbaum J, Vychytil A, Lhotta K, Prischl FC, Wiesholzer M, Machhold-Fabrizii V, Kopriva-Altfahrt G, Schwarz C, Balcke P, Oberbauer R, Kramar R, Konig P, Rudnicki M: Treatment with oral active vitamin D is associated with decreased risk of peritonitis and improved survival in patients on peritoneal dialysis. PLoS One 2013;8:e67836.

29 Su G, Liu Z, Qin X, Hong X, Liu X, Wen Z, Lindholm B, Carrero JJ, Johnson DW, Brusselaers N, Stalsby Lundborg C: Vitamin D deficiency and treatment versus risk of infection in end-stage renal disease patients under dialysis: a systematic review and meta-analysis. Nephrol Dial Transplant 2018; DOI:10.1093/ndt/ gfy216.

-30 Hsieh YP, Chang CC, Wen YK, Chiu PF, Yang Y: Predictors of peritonitis and the impact of peritonitis on clinical outcomes of continuous ambulatory peritoneal dialysis patients in Taiwan-10 years' experience in a single center. Perit Dial Int 2014;34:85-94.

-31 Szeto C: Peritoneal dialysis-related infection in the older population. Perit Dial Int 2015;35:659-662.

-32 Hsieh YP, Wang SC, Chang CC, Wen YK, Chiu PF, Yang Y: The negative impact of early peritonitis on continuous ambulatory peritoneal dialysis patients. Perit Dial Int 2014;34:627-635.

-33 Wang Z, Jiang L, Feng S, Yang L, Jiang S, Zhan Z, Song K, Shen H: Early peritonitis is an independent risk factor for mortality in elderly peritoneal dialysis patients. Kidney Blood Press Res 2015;40:298-305.

-34 Boudville N, Kemp A, Clayton P, Lim W, Badve SV, Hawley CM, McDonald SP, Wiggins KJ, Bannister KM, Brown FG, Johnson DW: Recent Peritonitis Associates with Mortality among Patients Treated with Peritoneal Dialysis. J Am Soc Nephrol 2012;23:1398-1405. 